\title{
Cooperative Coevolution with Two-Stage Decomposition for Large-Scale Global Optimization Problems
}

\author{
H. D. Yue $\mathbb{D}$ and Y. Sun $(\mathbb{D}$ \\ School of Computer, Electronics and Information, Guangxi University, Nanning, Guangxi, China \\ Correspondence should be addressed to Y. Sun; sunyu@gxu.edu.cn
}

Received 29 July 2021; Accepted 15 October 2021; Published 29 October 2021

Academic Editor: Shi Cheng

Copyright (C) 2021 H. D. Yue and Y. Sun. This is an open access article distributed under the Creative Commons Attribution License, which permits unrestricted use, distribution, and reproduction in any medium, provided the original work is properly cited.

Cooperative coevolution (CC) is an effective framework for solving large-scale global optimization (LSGO) problems. However, CC with static decomposition method is ineffective for fully nonseparable problems, and CC with dynamic decomposition method to decompose problems is computationally costly. Therefore, a two-stage decomposition (TSD) method is proposed in this paper to decompose LSGO problems using as few computational resources as possible. In the first stage, to decompose problems using low computational resources, a hybrid-pool differential grouping (HPDG) method is proposed, which contains a hybrid-pool-based detection structure (HPDS) and a unit vector-based perturbation (UVP) strategy. In the second stage, to decompose the fully nonseparable problems, a known information-based dynamic decomposition (KIDD) method is proposed. Analytical methods are used to demonstrate that HPDG has lower decomposition complexity compared to state-of-the-art static decomposition methods. Experiments show that CC with TSD is a competitive algorithm for solving LSGO problems.

\section{Introduction}

Large-scale global optimization (LSGO) problems are widespread optimization problems in scientific research and engineering applications [1-6]. In this paper, continuous LSGO problems with 1000 or greater decision variables are studied and solved.

There are two main challenges in solving LSGO problems: (1) dimensional catastrophe [7] and (2) traditional mathematical algorithms and evolutionary algorithms (EAs) with rapid performance degradation as the dimensionality grows [8-10]. There are two main approaches to solve difficulties. The first approach is to design or improve EAs with powerful exploration so that the EAs avoid getting trapped in local optima in large-scale solution space [11]. The second approach is the CC with problem decomposition, which adopts the idea of divide-and-conquer and is divided into two stages $[12,13]$, problem decomposition and problem optimization. Recently, CC has been used to solve a variety of problems [14-16]. The second approach is the one studied in this paper.
Decomposition methods decompose LSGO problems into multiple subproblems. CC with dynamic decomposition method decomposes the problems dynamically during the optimization stage, although dynamic decomposition methods are costly to decompose problems, which decompose fully nonseparable problems into multiple subproblems according to certain criteria [17]. The most typical dynamic decomposition method is random grouping [18], but the random grouping method is unable to make nearoptimal decomposition for many variables. Other dynamic decomposition methods such as delta grouping lose effectiveness for partially separable problems with more than one nonseparable subcomponent [19], and CCEA-AVP is computationally expensive and effective only when detecting linear variable correlations without nonlinear interdependency [20].

CC with static decomposition method decomposes the problem in the problem decomposition stage, and advanced static decomposition method can accurately decompose problems [21-23]. The key for static decomposition method is to correctly identify the relationships between variables. 
The differential grouping (DG) method [24] is an effective decomposition method for identifying relationships between variables; however, DG has the drawbacks of being sensitive to threshold and not being able to identify overlapping problems. The variant DG2 of DG effectively alleviates the drawbacks of DG, but DG2 is costly to compute adaptive threshold [25]. Mei et al. proposed a global differential grouping (GDG) to alleviate the sensitivity of DG to threshold by setting a global threshold [26]. Sun et al. proposed a recursive differential grouping (RDG) [27] and RDG's variants RDG2 [28] and RDG3 [29] to further improve the performance of the DG decomposition problems. The recursive interaction structure in RDG, which significantly improves the variable interaction efficiency and the complexity of the variable interaction structure, is $O(n \log n)$, where $n$ denotes the problem dimension. Recently, Yang et al. proposed an efficient recursive differential grouping (ERDG) method to avoid repeated interactions during variable interactions [30]. However, the number of function evaluations (FEs) used to decompose problems increases quickly when the problem dimension rises; and the CC with MRF-based decomposition mechanism [31] proposed by Sun et al. and the threelevel recursive differential grouping method [32] proposed by Bin et al. did not improve the ability of CC to solve nonseparable problems.

However, the static decomposition method aims to group the decision variables correctly, so CC with static decomposition encounters a dilemma in solving the fully nonseparable problems. To further reduce the decomposition complexity and alleviate the dilemma, the main work of this paper is as follows:

(1) We propose a decomposition method TSD in this paper.

(2) An improved differential grouping method, HPDG, is proposed. HPDG is a static decomposition method with a very low complexity. A new decomposition framework, hybrid-pool-based detection structure (HPDS), is proposed in this paper. The complexity of HPDS is $O(n \log m)$, where $n$ and $m$ denote the problem dimension and the number of subproblems, respectively.

(3) A unit vector-based perturbation (UVP) strategy is proposed to improve the decomposition accuracy of HPDG.

(4) A known information-based dynamic decomposition (KIDD) method is proposed to decompose the fully nonseparable problems for alleviating the dilemma of CC with static decomposition in solving LSGO problems.

In the remainder of this article, we first introduce the differential grouping, variable interaction structure, and CC framework in Section 2. Then we present the CC with TSD in detail in Section 3. Numerical experiments are carried out and comparisons with the state-of-the-art algorithms are made in Section 4. Section 5 concludes the work of this paper and plans the next work.

\section{Related Work}

2.1. Cooperative Coevolution with Decomposition Method. Cooperative coevolution plays an important role in the development of populations, especially for large size populations. In evolutionary computation, the effectiveness of $\mathrm{CC}$ is very dependent on the efficient dividing of population, which in the LSGO problems is problem decomposition [24, 33-35].

The steps for CC to solve LSGO problems are described in Algorithm 1 [36]. subgroup ${ }_{j}$ holds the indexes of the decision variables of the $j_{\text {th }}$ subgroup. In traditional CC framework, computational resources are equally distributed, and line 5 is not executed. First, the population is initialized and static decomposition method decomposes LSGO problems (lines 1-2). Then each subgroup is optimized separately and the population is updated. To improve the utilization of computational resources, contribution-based CC (CBCC) is proposed as described in line 5 [34, 37]. After optimizing each subgroup once separately, the subgroup with the largest contribution value is selected to optimize to the termination condition. To balance the exploitation and exploration, the subgroup with the largest contribution is selected by CBCC3 [38], which is evolved to the condition where the subgroup with the second largest contribution from the last optimization is larger than the subgroup with the largest current contribution.

\subsection{Decomposition Method}

2.2.1. Problem Decomposition Framework. Problem decomposition framework is the logical structure of problem decomposition adopted by decomposition methods, and the logical structure determines the computational complexity of decomposition methods. Problem decomposition framework can be categorized into two types: double-pool decomposition framework and single-pool decomposition framework [39]. The double-pool decomposition framework includes a detection pool and an update pool, the identification between variables is performed in detection pool, and the identified variables are stored in the update pool, as shown in Figure 1(a). The variable interaction structure of the decomposition methods based on differential grouping belongs to the double-pool decomposition framework [24, 27-29]. In the single-pool decomposition framework, the two pools are integrated into a single one, as shown in Figure 1(b), and the interactions of decision variables can be identified one by one. Experiments [39] show that singlepool decomposition framework is more conducive to identification of the variables than double-pool decomposition framework. In this paper, we combine the advantages of these two decomposition frameworks and propose a hybrid-pool decomposition framework.

2.2.2. Differential Grouping and Its Improvement. For differential functions, there are three relationships between decision variables: no relationship, direct relationship, and indirect relationship [24]. Based on these three relationships, 
(1) initialize (population);

(2) subgroups $\longleftarrow$ group (FunID, D);

(3) for cycles $\leq$ Maximum do

(4) Optimize each subgroup separately;

(5) Select a subgroup with heaviest contribution and optimize to termination;

(6) end for

(7) return fitness;

Algorithm 1: CC_with_decomposition (FuncID, D).

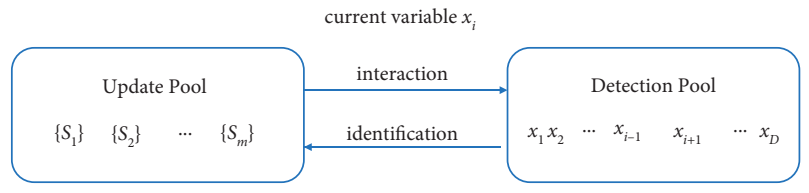

(a)

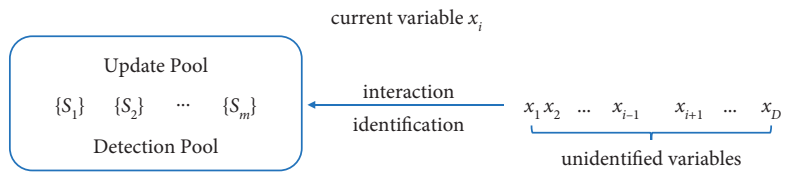

(b)

FIgURE 1: Decomposition framework. (a) Double-pool procedure. (b) Single-pool procedure.

three types of LSGO problems are constituted: full separable problem, partially separable problem, and full nonseparable problem, and the overlapping problem is a special type of partially separable problem. The primary task of problem decomposition is to identify the relationships between decision variables. For LSGO problem $f(X)$, the decision vector $X$ of $f(X)$ is divided into $m(1 \leq m \leq D)$ mutually exclusive subsets, as shown in equation (1), with $X_{i} \cap X_{j}=$ $\varnothing$ for any $X_{i}, X_{j} \in X$.

$$
\begin{aligned}
& X_{1}=\left\{x_{1}, x_{2}, \ldots, x_{p}\right\}, X_{2}=\left\{x_{p+1}, x_{p+2}, \ldots, x_{q}\right\}, \ldots, \\
& X_{m}=\left\{x_{k}, x_{k+1}, \ldots, x_{D}\right\} .
\end{aligned}
$$

DG decomposes problems by identifying the relationships between each pair of variables. Let the two variables identified be $x_{i}$ and $x_{j}$, if the partial derivative is

$$
\frac{\partial^{2} f(X)}{\partial x_{i} \partial x_{j}}=0(\text { or } \leq \varepsilon) \text {. }
$$

In the above equation, $\varepsilon$ is a very small positive real number parameter of DG used for determining whether two variables are related. Then $x_{i}$ is unrelated to $x_{j}$; otherwise $x_{i}$ is related to $x_{j}$. If the relationship between two variables is indirect, it is difficult for the DG to identify this relationship, as in the problem described by the following equation:

$$
f(X)=x_{1}+\left(x_{2}+x_{3}\right)^{2}+\left(x_{2}+x_{4}\right)^{2}
$$

where $x_{3}$ and $x_{4}$ are related through $x_{2}$ interacting with each other and DG cannot find the relationship between $x_{3}$ and $x_{4}$ because

$$
\frac{\partial^{2} f(X)}{\partial x_{3} \partial x_{4}}=0
$$

Problem decomposition by identifying the relationship between each pair of variables is inefficient. RDG significantly reduces the FEs by using a recursive detection structure in unidentified variables to complete problem decomposition. The theory of the identification for two sets of variables proved by Sun et al. is as follows [27]:

$$
f\left(x^{*}+l_{1} u_{1}+l_{2} u_{2}\right)-f\left(x^{*}+l_{2} u_{2}\right) \neq f\left(x^{*}+l_{1} u_{1}\right)-f\left(x^{*}\right) \text {. }
$$

Let $X_{1}, X_{2}$ be subsets of the decision vector $X$ of $f(X)$, let $u_{1}$ and $u_{2}$ be unit vectors of $X_{1}$ and $X_{2}$, respectively, and $x^{*}$ is a feasible solution, where $X_{1} \neq \varnothing, X_{2} \neq \varnothing, X_{1} \cap X_{2}=\varnothing$, and $l_{1}, l_{2}>0$. If some variables of $X_{1}$ and $X_{2}$ are related, inequality (7) holds. Conversely, $X_{1}$ and $X_{2}$ are not related to each other [27].

Based on RDG, ERDG uses interaction history information to complete problem decomposition and further reduces the FEs [27, 30]. However, RDG and ERDG apply perturbations to the variables by giving the same upper and lower bounds to the variables to be identified, so that the perturbation to identify all problems is fixed. The two changes of the fitness value are

$$
\begin{aligned}
& \Delta_{1}=f\left(X_{1, u b}\right)-f\left(X_{1, l b}\right), \\
& \Delta_{2}=f\left(X_{2 u, m b}\right)-f\left(X_{2 l, m b}\right),
\end{aligned}
$$

where $X_{1, u b}$ and $X_{1, l b}$ are candidate solutions that are given upper bounds and lower bounds, respectively, and $\mathrm{mb}$ denotes the mean of the upper bounds $u b$ and lower bounds $l b$. The variables belonging to $X_{2 u, m b}$ and $X_{2 l, m b}$ are assigned the same upper bounds and lower bounds as $X_{1}$, respectively, and the variables that do not belong to $X_{1}$ are assigned mb.

\section{Proposed Method}

3.1. Cooperative Coevolution with Two-Stage Decomposition. In this section, CC with TSD is proposed, which performs problem decomposition in the decomposition and optimization stages. In the decomposition stage, first the set of 
candidate solutions "population" is first initialized, and then HPDG groups the decision variables into different subgroups. In the optimization stage, KIDD dynamically decomposes the subgroups whose dimension is still high based on the decomposition information of HPDG, as described in Algorithm 2. The CC proposed in this paper can decompose different types of problems before and during the optimization stage, whereas the traditional CC decomposes the problems only before or during the optimization.

\subsection{Hybrid-Pool Differential Grouping}

3.2.1. Unit Vector-Based Perturbation. To determine whether two sets of variables are related, we apply perturbation to the sets. According to the IEEE 754 arithmetic [40], a large number of floating-point numbers affect the computational accuracy, and the HPD proposed in this paper uses the fixed-value perturbation described by equations (6) and (7) which affects the identification accuracy. We consider two types of problems divided in [41]:

Type 1: The problems whose fitness value changes a lot even when very small upper bounds and very great lower bounds are set to those decision variables.

Type 2: The problems whose fitness value changes slightly when very great upper bounds and very small lower bounds are set to those decision variables.

The two types of changes in fitness values listed above are absolute changes, and relative change of fitness values is defined as follows.

Definition 1. Let the differences between the maximum and minimum values of problem 1 and problem 2 be $\theta_{1}$ and $\theta_{2}$, and the changes in fitness values are $\vartheta_{1}$ and $\theta_{2}$ for the same perturbation done for both problems, respectively. If $\vartheta_{1} / \theta_{1}>\vartheta_{2} / \theta_{2}$, then the change in fitness values of problem 1 is greater than that of problem 2 .

Definition 1 means that the magnitude of the change in fitness is compared relatively. The perturbation is applied to the set of variables according to the following equation. According to equation (5), the UVP strategy is described in equations (8) and (9), where $l_{1}$ and $l_{2}$ are real numbers greater than 0 .

$$
\begin{array}{r}
\Delta_{1}=f\left(X \mid X_{1}=X_{1}+l_{1} u_{1}, X_{2}\right)-f\left(X \mid X_{1}, X_{2}\right), \\
\Delta_{2}=f\left(X \mid X_{1}=X_{1}+l_{1} u_{1}+l_{2} u_{2}, X_{2}=X_{2}+l_{2} u_{2}\right) \\
-f\left(X \mid X_{1}=X_{1}+l_{2} u_{2}, X_{2}=X_{2}+l_{2} u_{2}\right) .
\end{array}
$$

Subsets $X_{1}$ and $X_{2}$ of decision vector $X$ are initialized. Then, the unit vectors $u_{1}$ and $u_{2}$ of $X_{1}$ and $X_{2}$ are calculated separately, and the variables in the decision vectors $X_{1}$ and $X_{2}$ that do not belong to $X$ are assigned zero, and then the perturbations are applied to the currently identified subsets of variables, respectively.

3.2.2. Hybrid-Pool Decomposition Framework. The hybridpool decomposition framework is shown in Figure 2, where the detection pool and update pool are merged, all known
(1) Initialize population;

(2) subgroups « HPDG (FuncID, D);

(3) for $\mathrm{FE} s \leq$ Maximum do

(4) subgroups $\longleftarrow$ KIDD (subgroups);

(5) Traditional CC or contribution-based CC optimizes subgroups;

(6) end for

(7) return fitness; groupings as detection pool and update pool and unidentified variables as another detection pool with one unidentified subgroup. The decomposition produce of HPDS is as follows. The current variable $x_{i}$ identifies relationships with other variables in detection pool and update pool, as shown in Figure 2. The identification between variables is divided into two parts: one is the identification of $x_{i}$ with the unidentified variables set $V$, and the other is the identification of $x_{i}$ with each identified subgroup through binary interaction, as shown in lines 5-6 of Algorithm 3; the interaction details are shown in Algorithm 4. Then the results of the interaction between two parts are combined as the steps in lines 7-17 of Algorithm 3. Zero means that no variable set is related to $x_{i}$, and nonzero number means the index of the set related to $x_{i}$.

The steps of binary detection for the identification of the current variable $x_{i}$ with other variables are described in Algorithm 3. First, $x_{i}$ interacts with all known subgroups at one time; if $x_{i}$ is not related to all subgroups, algorithm exits; otherwise, the known subgroups will be divided into two equal-sized parts, and $x_{i}$ interacts with one part of the known subgroups, and if they are related, the subgroups continue recursion; otherwise, $x_{i}$ interacts with the other known subgroups recursively. Continue the above operations until the subgroup related to $x_{i}$ is identified.

\subsubsection{Decomposition Complexity Analysis of HPDG. The} detection complexity of the variable $x_{i}$ with known subgroups is shown in Figure 3. Let there be $m$ subgroups after the problem is decomposed, and the detection depth of $x_{i}$ is $p$. The part in curly bracket indicates the set of subgroups interacting with $x_{i}$. The number of subgroups $m$ in the $p$ th level can be expressed as $m=2^{p-1}$, so the detection depth $p=\log m+1$, and the decomposition complexity of HPDG of $n$-dimensional problem is $O(n \log m)$.

\subsection{Known Information-Based Dynamic Decomposition} Method. In this section, we propose the KIDD method for the limitation that the static decomposition method cannot decompose fully nonseparable problems. KIDD and HPDG together constitute the TSD method to improve the performance of CC solving LSGO problems. KIDD is detailed in Algorithm 5.

KIDD checks all subgroups' dimension decomposed by HPDG; if the subgroup's dimension exceeds $\operatorname{sub} D$, the 


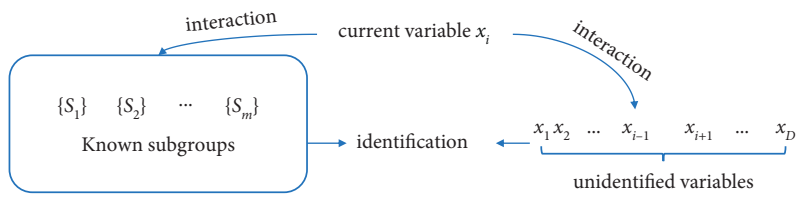

FIgURE 2: Hybrid-pool procedure.

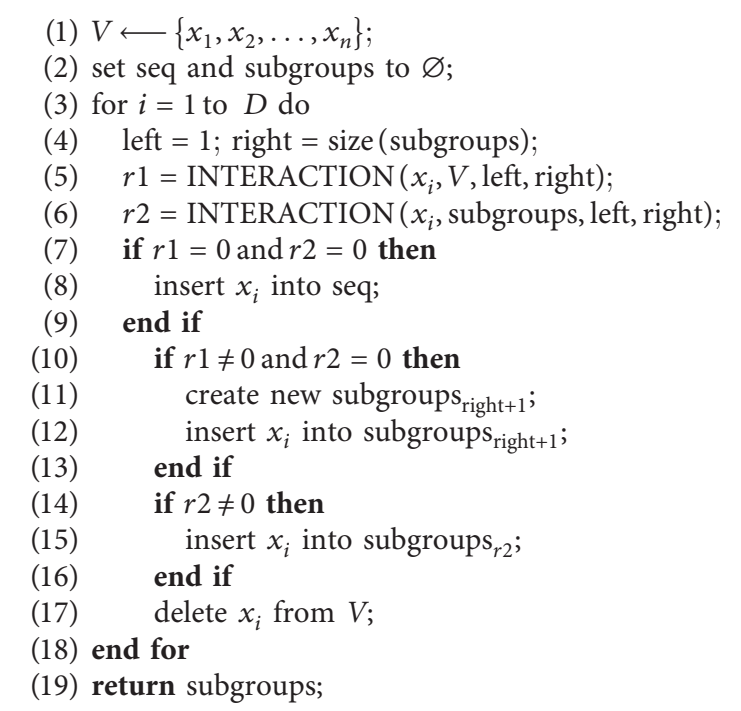

Algorithm 3: HPDG (FuncID, D).

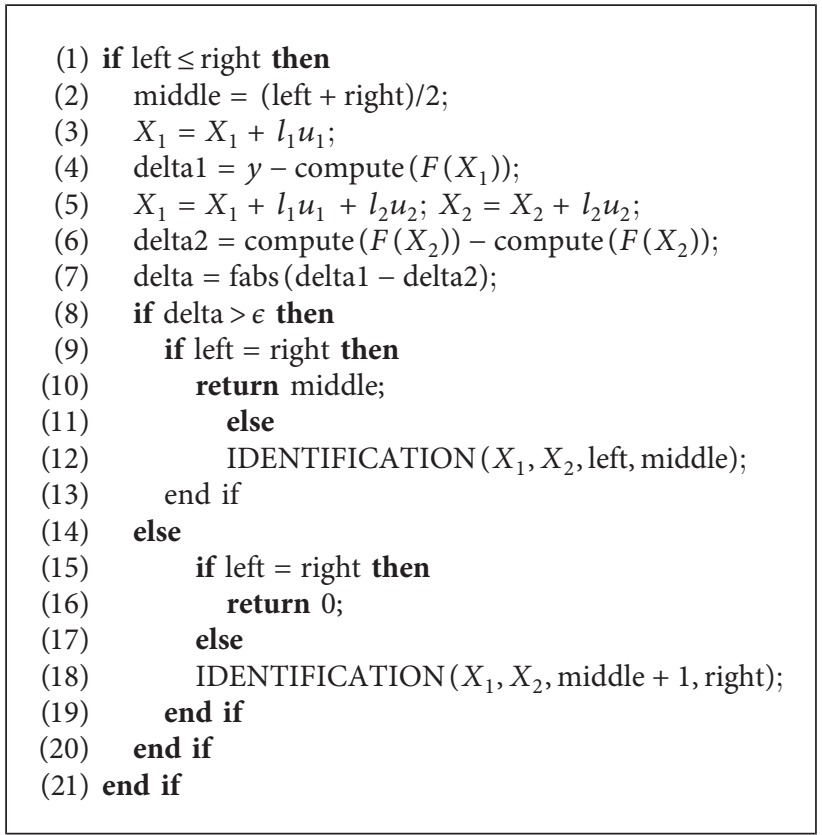

Algorithm 4: Interaction ( $X_{1}, X_{2}$, left, right).

subgroup is decomposed by random grouping [18] and update subgroups set. sub $D$ denotes subgroup dimension, and the value of subD is set according to the performance of the optimizer used by CC.

\section{Experiment Results and Discussion}

4.1. Experimental Methodology. The performances of the decomposition methods were compared on the IEEE 


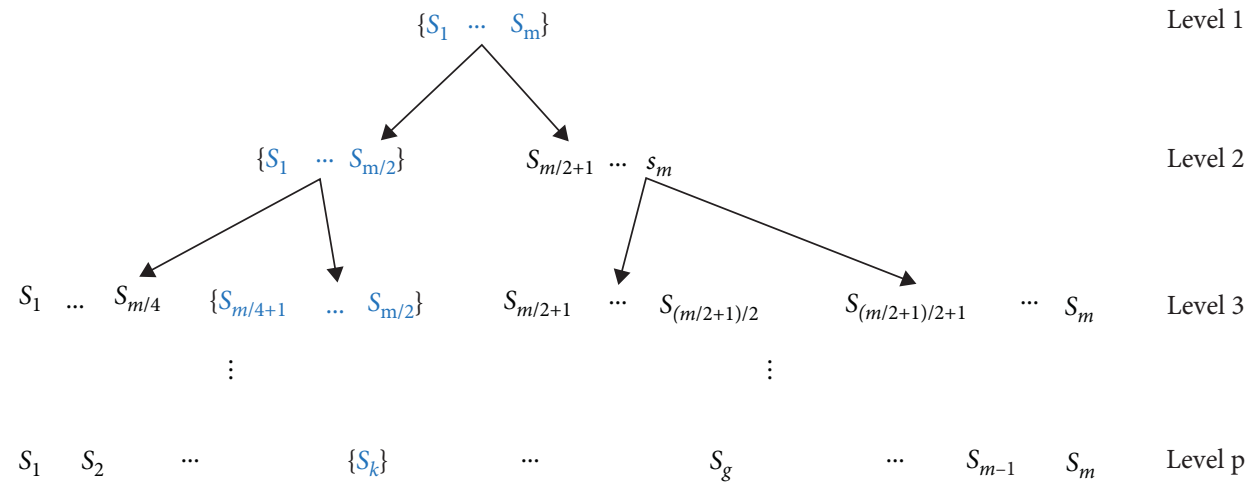

Figure 3: HPD detection depth analysis.

\author{
(1) while $i=1$ to size(subgroups) do \\ (2) if num $\left(\operatorname{subgroup}_{i}\right)>\operatorname{sub} D$ then \\ (3) random decomposition subgroup ; $_{i}$ \\ (4) update subgroups; \\ (5) end if \\ (6) end while \\ (7) return subgroups;
}

Algorithm 5: KIDD (subgroups).

CEC'2010 and CEC'2013 special sessions on large-scale global optimization $[42,43]$. The experiments in this paper were divided into two parts. In the first part, HPDG was compared with DG [24], DG2 [25], RDG [27], RDG2 [28], and ERDG [30] on problem decomposition. HPDG set $\varepsilon$ as RDG did, and the values of $\alpha$ used to calculate $\varepsilon$ are shown in Tables 1 and 2 .

Three evaluation metrics were selected to evaluate the performance of the decomposition methods:

(1) The decomposition accuracy is calculated as [30]; decision variables that are corelated to each other are grouped into the same subgroup and the variables that are not corelated to each other are grouped into different subgroups. In the experimental tables, "separable" and "non-sep" columns represent the decomposition accuracy of separable and nonseparable variables, respectively.

(2) The number of function evaluations (FEs) is used to decompose the problem $[42,43]$.

(3) In this paper, the growth rate of FEs is introduced to evaluate the performance of decomposition methods on high-dimensional problems, as in the following equation:

$$
\mathrm{gr}=\frac{\mathrm{hdFEs}-\mathrm{ldFEs}}{\mathrm{ldFEs}} \times 100 \% \text {, }
$$

where gr denotes the growth rate of FEs, and hdFEs and ldFEs denote the FEs of two different dimensional problems, respectively.
In the second part, TSD and other decomposition methods embedded in DECC were compared on problem optimization. Adaptive differential evolution with optional external archive (JADE) [44] was used to optimize the problem in optimization stage. FEs are set to $3 e+6$ as the termination condition for each run of the experiment [43]. The parameters of the decomposition methods and JADE were set as in their publications.

\subsection{Decomposition Comparison}

4.2.1. Comparison on Perturbation Strategy. In this section, we verify the effectiveness of UVP. As described in Table 3, HPDS with UVP correctly decomposes the four test functions $f 3, f 6, f 7$, and $f 10$ and improves the decomposition accuracy on $f 8$ and $f 11$ compared with HPDS with fixedvalue perturbation (FVP). That is because UVP applies perturbation of appropriate size when two sets of the variables interact, while the perturbation applied by FVP cannot satisfy the requirements of different problems.

4.2.2. Decomposition Results of HPDG and Other Decomposition Methods. Table 4 summarizes the decomposition results on CEC'2013 special sessions on large-scale global optimization. HPDG correctly decomposes all functions except $f 5, f 8, f 11, f 13$, and $f 15$. HPDG suffers from a flaw when detecting the indirect relationship between variables; if the shared variables are not grouped firstly, the indirect related variables cannot be grouped into the same subgroup. Although the number of functions correctly decomposed by HPDG is similar compared to DG2, RDG, and RDG2, HPDG uses fewer Fes; as shown in the last row of Table 3, HPDG correctly decomposes 7 functions using fewer FEs than other methods. HPDG uses the fewest FEs on 9 out of 15 functions; this is because HPDG has lower decomposition complexity compared to other methods.

4.2.3. Decomposition Results on High-Dimensional Functions. Since the structures of CEC'2013 special sessions on large-scale global optimization are fixed, the robustness of the decomposition methods is compared on extended high-dimensional CEC'2010 special sessions on large-scale 
TABLE 1: The values of $\alpha$ in CEC'2013 special sessions on large-scale global optimization.

\begin{tabular}{|c|c|c|c|c|c|c|c|c|c|c|}
\hline$F$ & $f 1$ & $f 2$ & f3 & $f 4-f 5$ & f6 & $f 7-f 8$ & f9 & $f 10$ & $f 11$ & $f 12-f 15$ \\
\hline$\alpha$ & $1.00 e-12$ & $1.00 e-10$ & $1.00 e-14$ & $1.00 e-12$ & $1.00 e-15$ & $1.00 e-12$ & $1.00 e-14$ & $1.00 e-15$ & $1.00 e-16$ & $1.00 e-12$ \\
\hline
\end{tabular}

TABLe 2: The values of $\alpha$ in CEC’2010 special sessions on large-scale global optimization.

\begin{tabular}{cccccc}
\hline$F$ & $f 1-f 2$ & $f 3$ & $f 4-f 5$ & $f 6$ & $f 7-f 20$ \\
\hline$\alpha$ & $1.00 e-12$ & $1.00 e-03$ & $1.00 e-12$ & $1.00 e-10$ & $1.00 e-20$ \\
\hline
\end{tabular}

TABLE 3: Decomposition results of HPDG with UVP and FVP on 1000-dimensional CEC'2013 special sessions on large-scale global optimization. For each function, the best values of FEs and decomposition accuracy among different decomposition methods are shown in bold, respectively.

\begin{tabular}{|c|c|c|c|c|c|c|}
\hline \multirow{2}{*}{$F$} & \multicolumn{3}{|c|}{ HPDS with UVP } & \multicolumn{3}{|c|}{ HPDS with FVP } \\
\hline & FEs & Separable & Non-sep & FEs & Separable & Non-sep \\
\hline$f 1$ & 3008 & $100.0 \%$ & - & 3008 & $100.0 \%$ & - \\
\hline$f 2$ & 3008 & $100.0 \%$ & - & 3008 & $100.0 \%$ & - \\
\hline$f 3$ & 3008 & $0.0 \%$ & - & 3008 & $100.0 \%$ & - \\
\hline$f 4$ & 6704 & $100.0 \%$ & $100.0 \%$ & 6704 & $100.0 \%$ & $100.0 \%$ \\
\hline$f 5$ & 8014 & $100.0 \%$ & $0.0 \%$ & 8014 & $100.0 \%$ & $0.0 \%$ \\
\hline$f 6$ & 10884 & $0.0 \%$ & $0.0 \%$ & 10884 & $100.0 \%$ & $100.0 \%$ \\
\hline$f 7$ & 6672 & $0.0 \%$ & $0.0 \%$ & 6672 & $100.0 \%$ & $100.0 \%$ \\
\hline f8 & 8865 & - & $0.0 \%$ & 9506 & - & $20.0 \%$ \\
\hline$f 9$ & 15367 & - & $100.0 \%$ & 13668 & - & $100.0 \%$ \\
\hline$f 10$ & 13988 & - & $0.0 \%$ & 14008 & - & $100.0 \%$ \\
\hline$f 11$ & 14298 & - & $0.0 \%$ & 14298 & - & $25.0 \%$ \\
\hline$f 12$ & 5008 & - & $100.0 \%$ & 5008 & - & $100.0 \%$ \\
\hline$f 13$ & 13731 & - & $0.0 \%$ & 13804 & - & $0.0 \%$ \\
\hline$f 14$ & 13737 & - & $0.0 \%$ & 13388 & - & $0.0 \%$ \\
\hline$f 15$ & 5008 & - & $100.0 \%$ & 5008 & - & $100.0 \%$ \\
\hline
\end{tabular}

global optimization. The extended high-dimensional CEC'2010 functions as the consistent set in the original CEC'2010 special sessions on large-scale global optimization [30].

Tables 5-9 summarize the decomposition results of the decomposition methods on 1000- to 5000-dimensional functions. Except for DG, each decomposition method has similar decomposition accuracy on the extended high-dimensional CEC'2010 functions, and all can decompose most of them correctly, but the FEs are significantly different. As the dimension grows, FEs used by DG and DG2 increase the most, RDG and RDG2 follow, and ERDG and HPDG are the best. That is because the complexity of DG and DG2 is $O\left(n^{2}\right)$ $[24,25]$, and the complexity of RDG and RDG2 is $O(n \log n)[27,28]$. Table 10 summarizes the growth rates of FEs used by RDG, ERDG, and HPDG, which are calculated by equation (10). The least growth of FEs is used by ERDG and HPDG; this is because ERDG uses the historical information of variable interactions to avoid examining some interrelationship between variables [30], and the complexity of HPDG is $O(n \log m)$, which is the lowest. The FEs used by HPDG and ERDG are comparable. As described in Table 10, the growth rate of FEs used by HPDG is slightly better than that of ERDG.
4.3. Optimization Results of CC with Decomposition Methods. Table 11 summarizes the optimization results of the decomposition methods being embedded in DECC [24]. The DECC-TSD proposed in this paper achieves the best optimization results on six functions. Compared with the stateof-the-art DECC-ERDG, DECC-TSD is more advantageous on the fully nonseparable function $f 15$. Therefore, DECCTSD alleviates the dilemma of CC with static decomposition in solving the fully nonseparable problems.

On fully separable functions $f 1$ and $f 2$, each decomposition method decomposes the problems using the same FEs, so they achieve the same results. Because HPDG uses the least FEs for decomposing $f 3$ and $f 6$, both HPDG and TSD achieve the best scores. On the partly separable function $f 13$, RDG and RDG2 achieve the best scores, because HPDG and ERDG do not decompose it correctly. The optimization results of DECC-TSD with grey background in Table 11 indicate better optimization grades compared to DECCHPDG. In the first stage of TSD, HPDG does not reduce the dimension of the fully nonseparable function $f 15$, and, in the second stage of TSD, KIDD detects that the dimension of $f 15$ is high, so KIDD dynamically decomposes $f 15$, and thus the optimization result of DECC-TSD is better than that of DECC-HPDG on f15. Similarly, the optimization result of 


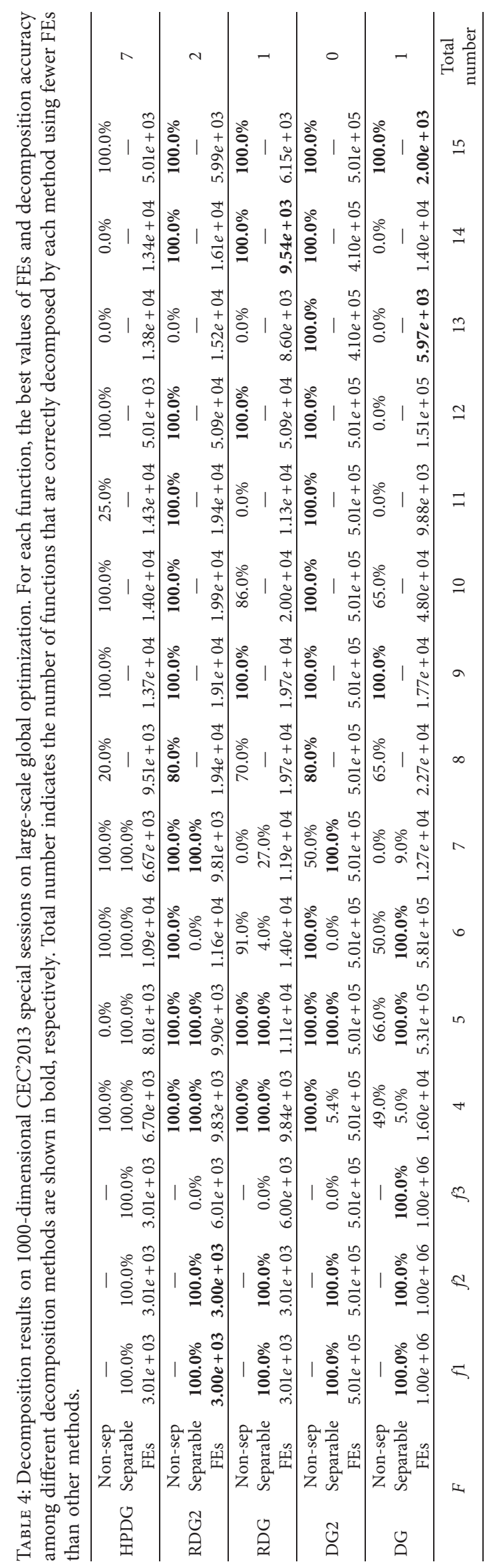




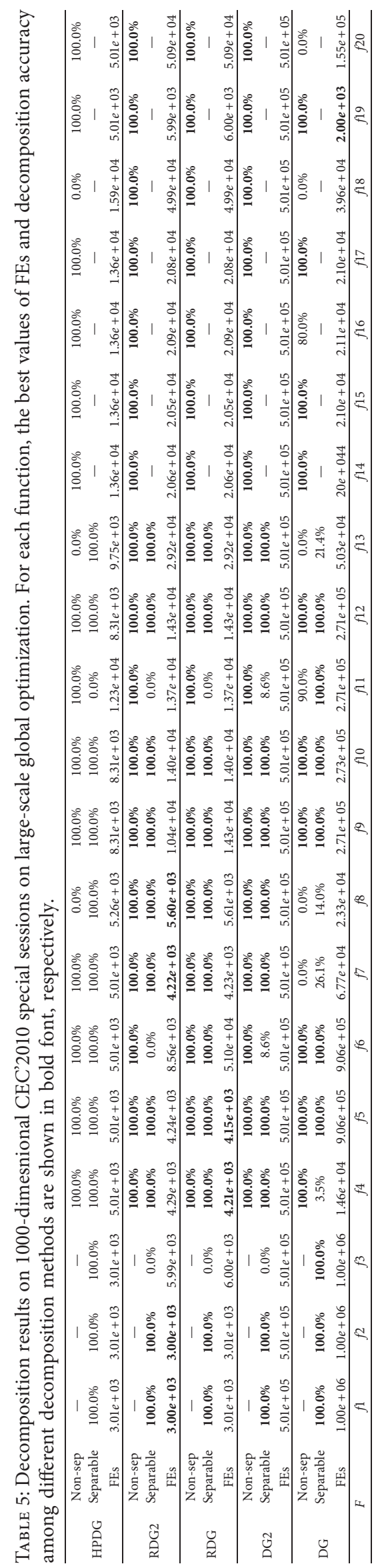




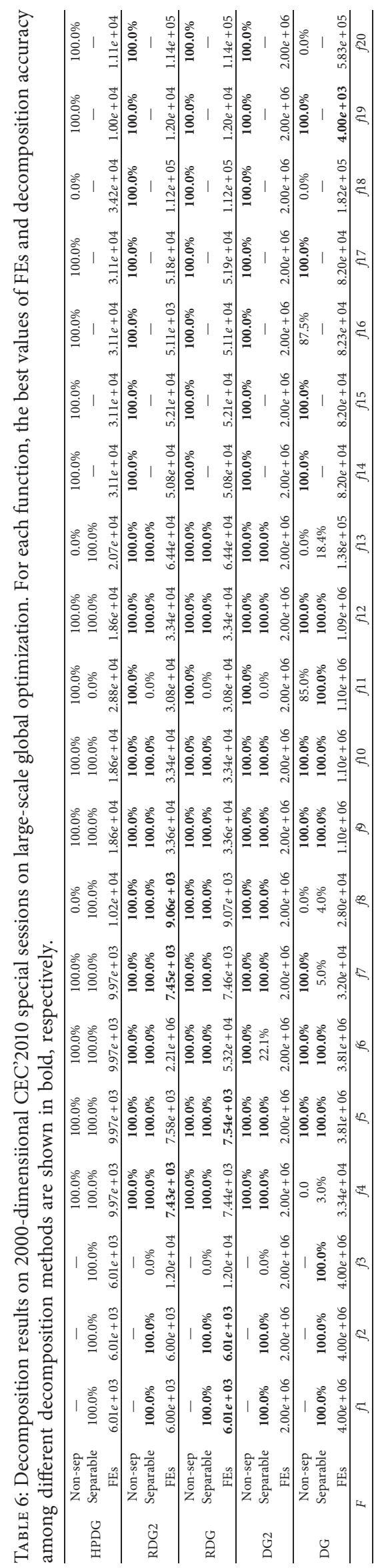









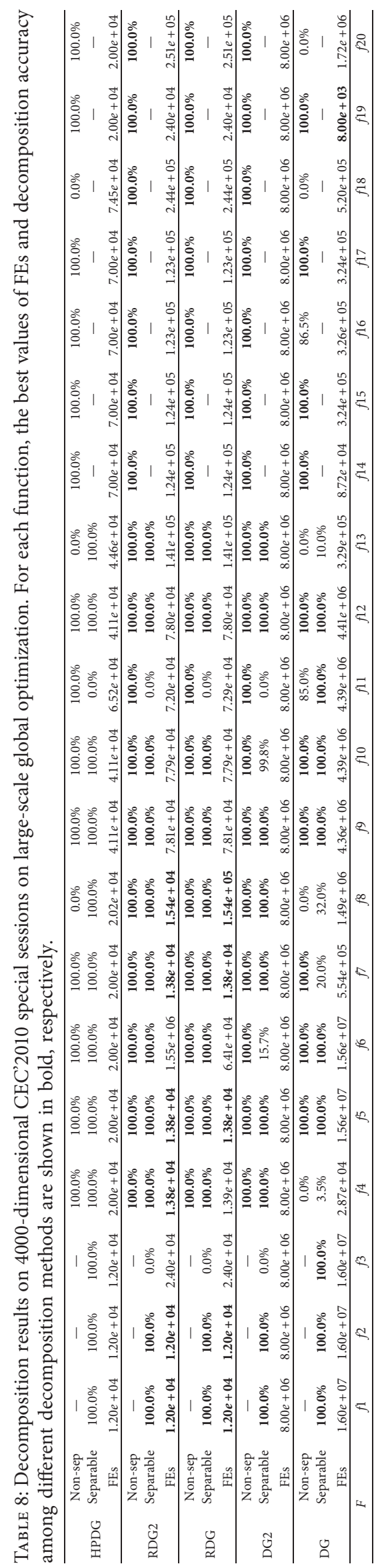




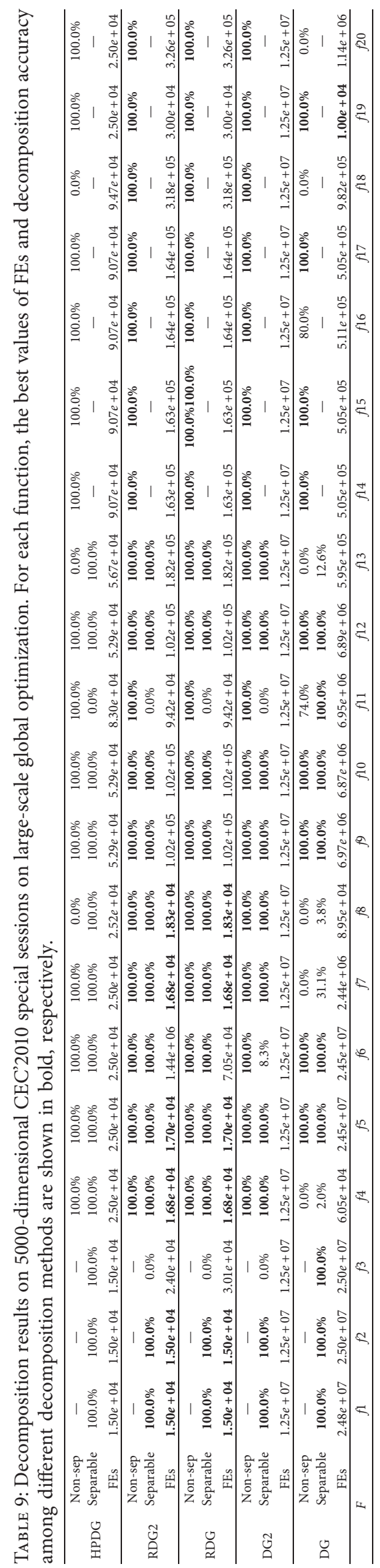


TABLE 10: The average growth rates of FEs of $f 1, f 2, f 4, f 5, f 7, f 10, f 12, f 13, f 14, f 15, f 16, f 17, f 18, f 19$, and $f 20$ on CEC'2010 special sessions on large-scale global optimization. The best growth rates of FEs are shown in bold font.

\begin{tabular}{lccc}
\hline Decomposition method & RDG (\%) & ERDG (\%) & HPDG (\%) \\
\hline$D=1000$ to $D=2000$ & 124.1 & 116. & $\mathbf{1 1 4 . 4}$ \\
$D=2000$ to $D=3000$ & 57.1 & $\mathbf{5 4 . 2}$ & $\mathbf{3 6 . 7}$ \\
$D=3000$ to $D=4000$ & 37.7 & 29.3 & 37.2 \\
$D=4000$ to $D=5000$ & 28.0 & $\mathbf{2 7 . 1}$ \\
\hline
\end{tabular}

TABLE 11: The optimization results on CEC'2013 special sessions on large-scale global optimization, $D=1000$. Average fitness values and deviations on CEC'2013 special sessions on large-scale global optimization over 25 independent results. The significantly best results are in bold (Wilcoxon test with home $p$ value correction, $\alpha=0.05$ ).

\begin{tabular}{|c|c|c|c|c|c|c|}
\hline$F$ & & DECC-RDG & DECC-RDG2 & DECC-ERDG & DECC-HPDG & DECC-TSD \\
\hline \multirow{2}{*}{$f 1$} & Mean & $3.66 E+11$ & $3.66 E+11$ & $3.41 E+11$ & $3.68 E+11$ & $3.69 E+11$ \\
\hline & Std & $1.24 E+10$ & $1.28 E+10$ & $1.22 E+10$ & $1.85 E+10$ & $1.36 E+10$ \\
\hline \multirow{2}{*}{$f 2$} & Mean & $1.34 E+05$ & $1.36 E+05$ & $1.44 E+05$ & $1.20 E+05$ & $1.21 E+05$ \\
\hline & Std & $4.43 E+03$ & $4.39 E+03$ & $4.40 E+03$ & $3.67 E+03$ & $3.55 E+03$ \\
\hline \multirow{2}{*}{$f 3$} & Mean & $3.42 E+01$ & $3.73 E+01$ & $3.41 E+01$ & $2.17 E+01$ & $2.16 E+01$ \\
\hline & Std & $5.31 E-03$ & $556 E-03$ & $5.41 E-03$ & $7.14 E-03$ & $6.84 E-03$ \\
\hline \multirow{2}{*}{$f 4$} & Mean & $4.53 E+12$ & $4.33 E+12$ & $4.13 E+12$ & $1.89 E+12$ & $3.33 E+12$ \\
\hline & Std & $1.98 E+11$ & $1.75 E+11$ & $1.27 E+11$ & $4.22 E+11$ & $5.36 E+11$ \\
\hline \multirow{2}{*}{$f 5$} & Mean & $5.21 E+07$ & $5.28 E+07$ & $1.21 E+07$ & $2.83 E+07$ & $3.24 E+07$ \\
\hline & Std & $4.32 E+06$ & $4.35 E+06$ & $2.12 E+06$ & $2.34 E+06$ & $2.97 E+06$ \\
\hline \multirow{2}{*}{$f 6$} & Mean & $1.10 E+06$ & $1.13 E+06$ & $9.98 E+06$ & $1.07 E+06$ & $1.07 E+06$ \\
\hline & Std & $1.21 E+02$ & $1.19 E+02$ & $1.31 E+02$ & $9.58 E+02$ & $7.87 E+02$ \\
\hline \multirow{2}{*}{$f 7$} & Mean & $6.12 E+11$ & $6.31 E+11$ & $1.12 E+11$ & $2.82 E+11$ & $1.25 E+11$ \\
\hline & Std & $3.01 E+11$ & $3.10 E+11$ & $2.47 E+11$ & $3.12 E+11$ & $4.07 E+10$ \\
\hline \multirow{2}{*}{$f 8$} & Mean & $5.11 E+15$ & $5.32 E+15$ & $5.11 E+14$ & $7.78 E+17$ & $8.14 E+17$ \\
\hline & Std & $1.99 E+15$ & $1.78 E+15$ & $1.54 E+14$ & $1.66 E+17$ & $2.11 E+17$ \\
\hline \multirow{2}{*}{ f9 } & Mean & $4.91 E+09$ & $4.93 E+09$ & $3.91 E+08$ & $1.70 E+09$ & $1.46 E+09$ \\
\hline & Std & $2.00 E+08$ & $2.05 E+08$ & $1.56 E+07$ & $1.57 E+08$ & $1.99 E+08$ \\
\hline \multirow{2}{*}{$f 10$} & Mean & $9.97 E+07$ & $9.88 E+07$ & $7.39 E+06$ & $9.58 E+07$ & $9.58 E+07$ \\
\hline & Std & $2.51 E+06$ & $2.43 E+06$ & $1.76 E+05$ & $2.31 E+05$ & $2.56 E+05$ \\
\hline \multirow{2}{*}{$f 11$} & Mean & $4.99 E+14$ & $4.97 E+08$ & $6.29 E+07$ & $1.09 E+14$ & $2.28 E+12$ \\
\hline & Std & $1.21 E+14$ & $1.36 E+08$ & $2.87 E+07$ & $1.23 E+14$ & $1.13 E+12$ \\
\hline \multirow{2}{*}{$f 12$} & Mean & $7.78 E+11$ & $7.86 E+11$ & $6.47 E+11$ & $8.70 E+11$ & $1.66 E+11$ \\
\hline & Std & $3.18 E+10$ & $3.27 E+10$ & $1.63 E+10$ & $4.64 E+10$ & $1.23 E+10$ \\
\hline \multirow{2}{*}{$f 13$} & Mean & $3.45 E+09$ & $3.58 E+09$ & $1.53 E+14$ & $1.87 E+16$ & $1.41 E+14$ \\
\hline & Std & $7.01 E+08$ & $7.18 E+08$ & $4.53 E+08$ & $1.84 E+16$ & $1.99 E+14$ \\
\hline \multirow{2}{*}{$f 14$} & Mean & $2.53 E+09$ & $2.43 E+09$ & $1.78 E+09$ & $1.25 E+15$ & $6.99 E+13$ \\
\hline & Std & $1.59 E+09$ & $1.35 E+09$ & $5.83 E+08$ & $7.00 E+14$ & $2.05 E+13$ \\
\hline \multirow[b]{2}{*}{$f 15$} & Mean & $9.09 E+13$ & $9.09 E+13$ & $7.08 E+13$ & $8.61 E+13$ & $2.91 E+11$ \\
\hline & Std & $2.49 E+13$ & $2.50 E+13$ & $2.50 E+13$ & $2.39 E+13$ & $2.81 E+11$ \\
\hline
\end{tabular}

DECC-TSD on $f 11$ is better than that of DECC-HPDG. Although HPDG does not decompose $f 12$ and $f 13$ correctly, KIDD performs an effective dimension reduction of the subgroups in the optimization stage, so DECC-TSD has better grades.

\section{Conclusions}

In this paper, we propose a TSD to improve the performance of CC in solving LSGO problems. In the first stage of TSD, HPDG is proposed, which provides a variable detection structure HPDS with very low complexity to decompose problems, and the UVP is proposed for HPDS to improve the decomposition accuracy. HPDG further reduces the FEs used to decompose the LSGO problems. In the second stage of TSD, KIDD decomposes the subgroups with high dimension based on the decomposition information of HPDG. TSD alleviates the dilemma of CC with static decomposition in solving fully nonseparable problems and reducing the computational cost of decomposition methods.

In future work, we plan to further improve the decomposition accuracy of HPDG and research new algorithms to solve fully nonseparable LSGO problems. 


\section{Data Availability}

The algorithms proposed in this paper are validated on two test suites with benchmark functions described in literatures [42] and [43].

\section{Conflicts of Interest}

The authors declare that they have no conflicts of interest.

\section{Acknowledgments}

This work was supported by the National Natural Science Foundation of China (Grants nos. 61763002 and 62072124), Guangxi Major Projects of Science and Technology (Grant no. 2020AA21077021), and Foundation of Guangxi Experiment Center of Information Science (Grant no. KF1401).

\section{References}

[1] Q. Yang, W.-N. Chen, T. Gu et al., "Segment-based predominant learning swarm optimizer for large-scale optimization," IEEE Transactions on Cybernetics, vol. 47, no. 9, pp. 2896-2910, 2017.

[2] R. Paz, E. Pei, M. Monzón, F. Ortega, and L. Suárez, "Lightweight parametric design optimization for 4D printed parts," Integrated Computer-Aided Engineering, vol. 24, no. 3, pp. 225-240, 2017.

[3] V. L. S. Silva, A. A. Emerick, P. Couto, and J. L. D. Alves, "History matching and production optimization under uncertainties - Application of closed-loop reservoir management," Journal of Petroleum Science and Engineering, vol. 157, pp. 860-874, 2017.

[4] A. Song, W.-N. Chen, T. Gu, H. Yuan, S. Kwong, and J. Zhang, "Distributed virtual network embedding system with historical archives and set-based particle swarm optimization," IEEE Transactions on Systems, Man, and Cybernetics: Systems, vol. 51, no. 2, pp. 927-942, 2021.

[5] R. Tang, X. Li, and J. Lai, "A novel optimal energy-management strategy for a maritime hybrid energy system based on large-scale global optimization," Applied Energy, vol. 228, pp. 254-264, 2018.

[6] L. Ma, S. Cheng, and Y. Shi, "Enhancing learning efficiency of brain storm optimization via orthogonal learning design," IEEE Transactions on Systems, Man, and Cybernetics: Systems, vol. 51, no. 11, pp. 6723-6742, 2021.

[7] Y. Sun, Y. Li, Y. Yang, and H. Yue, "Differential evolution algorithm with population knowledge fusion strategy for image registration," Complex \& Intelligent Systems, pp. 1-16, 2021.

[8] X. Wu, Y. Wang, J. Liu, and N. Fan, “A new hybrid algorithm for solving large scale global optimization problems," IEEE Access, vol. 7, pp. 103354-103364, 2019.

[9] S. Mahdavi, M. E. Shiri, and S. Rahnamayan, "Metaheuristics in large-scale global continues optimization: a survey," Information Sciences, vol. 295, pp. 407-428, 2015.

[10] S. Cheng, L. Ma, H. Lu, X. Lei, and Y. Shi, "Evolutionary computation for solving search-based data analytics problems," Artificial Intelligence Review, vol. 54, no. 2, pp. 1321-1348, 2020.

[11] H. Liu, Y. Wang, L. Liu, and X. Li, "A two phase hybrid algorithm with a new decomposition method for large scale optimization," Integrated Computer-Aided Engineering, vol. 25, no. 4, pp. 349-367, 2018.

[12] H. Liu, Y. Wang, X. Liu, and S. Guan, "Empirical study of effect of grouping strategies for large scale optimization," in Proceedings of the 2016 International Joint Conference on Neural Networks (IJCNN), pp. 3433-3439, Vancouver, British Columbia, Canada, July 2016.

[13] D. Yazdani, M. N. Omidvar, J. Branke, T. T. Nguyen, and $\mathrm{X}$. Yao, "Scaling up dynamic optimization problems: a divideand-conquer approach," IEEE Transactions on Evolutionary Computation, vol. 24, no. 1, pp. 1-15, 2020.

[14] A. Vakhnin and E. Sopov, "Investigation of improved cooperative coevolution for large-scale global optimization problems," Algorithms, vol. 14, no. 5, p. 146, 2021.

[15] J. Liang, R. Liu, K. Yu, and B. Qu, "Dynamic multi-swarm particle swarm optimization with cooperative coevolution for large scale global optimization," Journal of Software, vol. 29, no. 9, pp. 2595-2605, 2018.

[16] J.-R. Jian, Z.-H. Zhan, and J. Zhang, "Large-scale evolutionary optimization: a survey and experimental comparative study," International Journal of Machine Learning and Cybernetics, vol. 11, no. 3, pp. 729-745, 2020.

[17] X.-Y. Zhang, Y.-J. Gong, Y. Lin, J. Zhang, S. Kwong, and J. Zhang, "Dynamic cooperative coevolution for large scale optimization," IEEE Transactions on Evolutionary Computation, vol. 23, no. 6, pp. 935-948, 2019.

[18] M. N. Omidvar, X. Li, Z. Yang, and X. Yao, "Cooperative coevolution for large scale optimization through more frequent random grouping," in Proceedings of the 2010 IEEE Congress on Evolutionary Computation (CEC), pp. 1-8, Barcelona, Spain, July 2010.

[19] M. N. Omidvar, X. Li, and X. Yao, "Cooperative co-evolution with delta grouping for large scale non-separable function optimization," in Proceedings of the 2010 IEEE Congress on Evolutionary Computation (CEC), pp. 1-8, Barcelona, Spain, July 2010.

[20] T. Ray and X. Yao, "A cooperative coevolutionary algorithm with correlation based adaptive variable partitioning," in Proceedings of the 2009 IEEE Congress on Evolutionary Computation (CEC), pp. 983-989, Trondheim, Norway, May 2009.

[21] L. Li, W. Fang, Y. Mei, and Q. Wang, "Cooperative coevolution for large-scale global optimization based on fuzzy decomposition," Soft Computing, vol. 25, no. 5, pp. 3593-3608, 2021.

[22] X. Ma, X. Li, Q. Zhang et al., "A survey on cooperative coevolutionary algorithms," IEEE Transactions on Evolutionary Computation, vol. 23, no. 3, pp. 421-441, 2019.

[23] X.-M. Hu, F.-L. He, W.-N. Chen, and J. Zhang, "Cooperation coevolution with fast interdependency identification for large scale optimization," Information Sciences, vol. 381, pp. 142160, 2017

[24] M. N. Omidvar, X. Li, Y. Mei, and X. Yao, "Cooperative coevolution with differential grouping for large scale optimization," IEEE Transactions on Evolutionary Computation, vol. 18, no. 3, pp. 378-393, 2014.

[25] M. N. Omidvar, M. Yang, Y. Mei, X. Li, and X. Yao, "DG2: a faster and more accurate differential grouping for large-scale black-box optimization," IEEE Transactions on Evolutionary Computation, vol. 21, no. 6, pp. 929-942, 2017.

[26] Y. Mei, M. N. Omidvar, X. Li, and X. Yao, "A competitive divide-and-conquer algorithm for unconstrained large-scale black-box optimization," ACM Transactions on Mathematical Software, vol. 42, no. 2, pp. 1-24, 2016. 
[27] Y. Sun, M. Kirley, and S. K. Halgamuge, "A recursive decomposition method for large scale continuous optimization," IEEE Transactions on Evolutionary Computation, vol. 22, no. 5, pp. 647-661, 2017.

[28] Y. Sun, M. Omidvar, M. Kirley, and X. Li, “Adaptive threshold parameter estimation with recursive differential grouping for problem decomposition," in Proceedings of the 2018 the Genetic and Evolutionary Computation Conference, pp. 889-896, Kyoto, Japan, July 2018.

[29] Y. Sun, X. Li, A. Ernst, and M. N. Omidvar, "Decomposition for large-scale optimization problems with overlapping components," in Proceedings of the 2019 IEEE Congress on Evolutionary Computation (CEC), pp. 326-333, Wellington, New Zealand, June 2019.

[30] M. Yang, A. Zhou, C. Li, and X. Yao, "An efficient recursive differential grouping for large-scale continuous problems," IEEE Transactions on Evolutionary Computation, vol. 25, no. 1, pp. 159-171, 2021.

[31] L. Sun, L. Lin, M. Gen, and H. Li, "Co-evolutionary algorithm with MRF-based decomposition mechanism for flexible scheduling with uncertainty," in Proceedings of the 2019 IEEE International Conference on Smart Manufacturing, Industrial \& Logistics Engineering (SMILE), pp. 230-234, Hangzhou, China, April 2019.

[32] H.-B. Xu, F. Li, and H. Shen, "A three-level recursive differential grouping method for large-scale continuous optimization," IEEE Access, vol. 8, pp. 141946-141957, 2020.

[33] Y. Wang, H. Liu, F. Wei, T. Zong, and X. Li, "Cooperative coevolution with formula-based variable grouping for largescale global optimization," Evolutionary Computation, vol. 26, no. 4, pp. 569-596, 2018.

[34] M. Yang, M. N. Omidvar, C. Li et al., "Efficient resource allocation in cooperative co-evolution for large-scale global optimization," IEEE Transactions on Evolutionary Computation, vol. 21, no. 4, pp. 493-505, 2017.

[35] M. Yang, A. Zhou, C. Li, J. Guan, and X. Yan, "CCFR2: a more efficient cooperative co-evolutionary framework for largescale global optimization," Information Sciences, vol. 512, pp. 64-79, 2020.

[36] M. N. Omidvar, X. Li, and X. Yao, "Smart use of computational resources based on contribution for cooperative coevolutionary algorithms," in Proceedings of the 13th Annual Conference on Genetic and Evolutionary Computation, pp. 1115-1122, Dublin, Ireland, July 2011.

[37] B. Kazimipour, M. N. Omidvar, X. Li, and A. K. Qin, “A sensitivity analysis of contribution-based cooperative coevolutionary algorithms," in Proceedings of the 2015 IEEE Congress on Evolutionary Computation (CEC), pp. 417-424, Sendai, Japan, May 2015.

[38] M. N. Omidvar, B. Kazimipour, X. Li, and X. Yao, "CBCC3-a contribution-based cooperative co-evolutionary algorithm with improved exploration/exploitation balance," in Proceedings of the 2016 IEEE Congress on Evolutionary Computation (CEC), pp. 3541-3548, Vancouver, British Columbia, Canada, July 2016.

[39] X. Xue, K. Zhang, R. Li et al., "A topology-based single-pool decomposition framework for large-scale global optimization," Applied Soft Computing, vol. 92, Article ID 106295, 2020.

[40] IEEE, Std 754-2019 IEEE Standard for Floating-Point Arithmetic, IEEE, Piscataway, NJ, USA, pp. 1-84, 2019.

[41] H. Liu, Y. Wang, and N. Fan, "A hybrid deep grouping algorithm for large scale global optimization," IEEE
Transactions on Evolutionary Computation, vol. 24, no. 6, pp. 1112-1124, 2020.

[42] K. Tang, X. Yao, P. Suganthan, Z. Yang, and T. Weise, "Benchmark functions for the CEC'2010 special session and competition on large scale global optimization," Tech. Report, pp. 1-23, Nature Inspired Computation and Application. Laboratory, USTC, Hefei, China, 2010.

[43] X. Li, K. Tang, M. N. Omidvar, Z. Yang, and K. Qin, "Benchmark functions for the CEC 2013 special session and competition on large-scale global optimization," Gene, vol. 7, no. 33, p. 8, 2013.

[44] J. Zhang and A. C. Sanderson, "JADE: adaptive differential evolution with optional external archive," IEEE Transactions on Evolutionary Computation, vol. 13, no. 5, pp. 945-958, 2009. 